Pacific Journal of Mathematics

ON THE APPROXIMATION OF A FUNCTION OF SEVERE
VARIABLES BY THE SUM OF FUNCTIONS OF FEWER Stephen P.L. Diliberto and ERnst Gabon Straus 


\title{
ON THE APPROXIMATION OF A FUNCTION OF SEVERAL VARIABLES BY THE SUM OF FUNCTIONS OF FEWER VARIABLES
}

\author{
S. P. Diliberto and E. G. Straus
}

1. The problems. Let $R$ denote the unit square $0 \leq x \leq 1,0 \leq y \leq 1$, and $C_{R}$ the space of all continuous real-valued functions $z$ defined on $R$, with norm $\|z\|$ defined by $\|z\|=\max (x, y) \epsilon R|z|$. Let $I_{x}$ and $I_{y}$ denote respectively the unit intervals $0 \leq x \leq 1$ and $0 \leq y \leq 1$; and let $C_{x}$ and $C_{y}$ denote respectively the classes of all continuous functions on $I_{x}$ and $I_{y}$. By an obvious identification $C_{x}$ and $C_{y}$ may be considered as subsets of $C_{R}$. Let $C_{S}$ denote the subset of $C_{R}$ composed of all functions $z \in C_{R}$ such that $z=f+g$ where $f \in C_{x}$ and $g \in C_{y} ; C_{S}$ is closed (under the above norm).

For $z \in C_{R}$, define the functional $\mu[z]$ by

$$
\mu[z]=\operatorname{dist}\left[z, C_{S}\right]=\inf _{w \in C_{s}}\|z-w\| \text {. }
$$

The following problem was posed by The RAND Corporation.

Problem (A): Given $z \in C_{R}$ and $\epsilon>0$, give a method for evaluating $\mu[z]$ to within $\epsilon .^{*}$

Problem (B): Given $z \in C_{R}$ and $\epsilon>0$, give a method for constructing functions $f \in C_{x}$ and $g \in C_{y}$ such that

$$
\|z-f-g\| \leq \mu[z]+\epsilon .
$$

It is our purpose in the present note to solve these problems and to establish certain generalizations.

Received November 7, 1950.

* Actually, this differs somewhat from the problem as formulated by RAND, which was : Given $z$ and $\delta$, give a method for determining whether $\mu[z]<\delta$. This is in all probability unsolvable when $\mu[z]=\delta$, since any computation of $\mu[z]$ which can be carried out in a finite number of steps will, in general, yield only an approximation.

Pacific J. Math. 1 (1951), 195-210. 
2. The role of the minimizing sequence. We shall now define a few terms by means of which our procedure can be outlined conveniently.

We shall say that two functions $z$ and $\bar{z}$ in $C_{R}$ are equivalent if $z-\bar{z} \in C_{S}$, and shall denote the equivalence of $z$ and $\bar{z}$ by $z \sim \bar{z}$. Clearly, $z \sim \bar{z}$ implies $\mu[z]=\mu[\bar{z}]$.

According to the definition of $\mu[z]$, there exists a sequence of functions $\left\{w_{i}\right\}, w_{i} \in C_{S}$, such that

$$
\left\|z-w_{i}\right\| \rightarrow \mu[z]
$$

Let us define $z_{i}=z-w_{i}$; then $z_{i} \sim z$ and $\left\|z_{i}\right\| \rightarrow \mu[z]$. We shall call a sequence $\left\{z_{i}\right\}, z_{i} \in C_{R}$, a minimizing sequence for $z$ if $z_{i} \sim z$ for all $i$ and $\left\|z_{i}\right\| \rightarrow \mu[z]$.

Clearly, both of the proposed problems will be solved once one has constructed a minimizing sequence.*

We shall introduce a "leveling process," which when applied to $z$ and then iterated will produce a sequence of functions $\left\{z_{i}\right\}$ with the properties (1) $z_{i} \sim z$ and (2) $\left\|z_{i}\right\| \geq\left\|z_{i+1}\right\|$ for all $i$. Properties (1) and (2) imply

$$
\lim _{i \rightarrow \infty}\left\|z_{i}\right\|=M \geq \mu[z]
$$

That $M=\mu[z]$, that is, that our "leveling sequence" is in fact a minimizing sequence, is the principal result of this paper.

This will be established by a "pincers" argument to obtain convergence$\mu[z]$ is simultaneously approximated from above and below: For each path in the class of admissible paths $L$ (defined below) we shall define a functional $\pi_{l}[z]$, over $C_{R}$, with the important property $\pi_{l}[z] \leq \mu[z]$. Let

$$
\sup _{l \in L}\left|\pi_{l}[z]\right|=\pi[z] \text {. }
$$

Then

$$
\pi[z] \leq \mu[z] \leq M
$$

* Given a sequence of real numbers $a_{i} \rightarrow a$, let us call the integer-valued function $N(\epsilon)$ of the real variable $\epsilon$, defined for $\epsilon \geq 0$, a modulus of convergence for the sequence $a_{i}$, if $i \geq N(\epsilon)$ implies $\left|a_{i}-a\right|<\epsilon$.

While a method for constructing a minimizing sequence answers the questions, the finiteness of the procedure is satisfactory only when one has an estimate for the modulus of convergence. This will be discussed at the end of this paper. 
Our proof is accomplished by showing that $\pi[z]=M$, thus implying also that $\pi[z]=\mu[z]=M$.

3. The main theorem. We shall say that a closed polygonal line is permissible if it lies entirely within the square $0 \leq x \leq 1,0 \leq y \leq 1$, and if each of its sides is parallel either to the $x$ - or to the $y$-axis.

We enumerate the vertices of a permissible line by $\left(x_{j}, y_{j}\right), j=1,2, \cdots$, where

$$
x_{2 k-1}=x_{2 k}, \quad y_{2 k+1}=y_{2 k}, \quad x_{k+2 n}=x_{k}, \quad y_{k+2 n}=y_{k} ; \quad k=1,2, \cdots .
$$

To each permissible polygonal line $l$ we can associate a functional $\pi_{l}[z]$ with

$$
\pi_{l}[z]=\frac{1}{2 n} \sum_{j=1}^{2 n}(-1)^{j} z\left(x_{j}, y_{j}\right)
$$

Lemma 1. If $z \sim \bar{z}$, then $\pi_{l}[z]=\pi_{l}[\bar{z}]$.

Proof. Let $\bar{z}(x, y)=z(x, y)+g(x)+h(y)$; then

$$
\sum_{j=1}^{2 n}(-1)^{j} \bar{z}\left(x_{j}, y_{j}\right)=\sum_{j=1}^{2 n}(-1)^{j} z\left(x_{j}, y_{j}\right)+\sum_{j=1}^{2 n}(-1)^{j} g\left(x_{j}\right)+\sum_{j=1}^{2 n}(-1)^{j} h\left(y_{j}\right) .
$$

But

$$
\begin{aligned}
& \sum_{j=1}^{2 n}(-1)^{j} g\left(x_{j}\right)=-\sum_{k=1}^{n} g\left(x_{2 k-1}\right)+\sum_{k=1}^{n} g\left(x_{2 k}\right)=0, \\
& \sum_{j=1}^{2 n}(-1)^{j} h\left(y_{j}\right)=-\sum_{k=1}^{n} h\left(y_{2 k+1}\right)+\sum_{k=1}^{n} h\left(y_{2 k}\right)=0 .
\end{aligned}
$$

Hence

$$
\sum_{j=1}^{2 n}(-1)^{j} \bar{z}\left(x_{j}, y_{j}\right)=\sum_{j=1}^{2 n}(-1)^{j} z\left(x_{j}, y_{j}\right) ;
$$

that is, $\pi_{l}[\bar{z}]=\pi_{l}[z]$.

We remark that these invariants (under equivalence) $\pi_{l}[z]$ form a complete set of invariants. That is to say: If $\pi_{l}[\bar{z}]=\pi_{l}[z]$ for all permissible lines, then 
$z \sim \bar{z}$. In fact the $\pi_{l}$ based on rectangles alone form a complete set of invariants. In order to relate $\mu$ with the $\pi_{l}$ we prove the following result.

Lemma 2. The functional $\mu[z]$ satisfies $\mu[z] \geq\left|\pi_{l}[z]\right|$ for all permissible lines.

Proof. If we had $\mu[z]<\left|\pi_{l}[z]\right|$ then there would exist a function $\bar{z} \sim z$ such that $\|\bar{z}\|<\left|\pi_{l}[z]\right|$ and hence :

$$
\begin{aligned}
\left|\pi_{l}[z]\right| & =\left|\pi_{l}[\bar{z}]\right|=\frac{1}{2 n}\left|\sum_{j=1}^{2 n}(-1)^{j} \bar{z}\left(x_{j}, y_{j}\right)\right| \\
& \leq \frac{1}{2 n} \sum_{j=1}^{2 n}\left|\bar{z}\left(x_{j}, y_{j}\right)\right| \leq\|\bar{z}\|<\left|\pi_{l}[z]\right|,
\end{aligned}
$$

which is a contradiction.

Problem A will be solved once we establish the following theorem.

Тнеовем 1. The functional $\mu[z]$ satisfies $\mu[z]=\sup \left|\pi_{l}[z]\right|=\pi[z]$, where the sup is taken over all permissible lines.

As a preliminary to the proof of this the orem we introduce the following leveling process :

Given $z \in C_{R}$, we define the sequences of functions $z_{n}(x, y), g_{n}(x), h_{n}(y)$ $(n=1,2, \cdots)$ by the relations :

$$
\begin{aligned}
z_{1} & =z, \quad z_{2 n}=z_{2 n-1}-g_{n}, \quad z_{2 n+1}=z_{2 n}-h_{n}, \\
g_{n}(x) & =\frac{1}{2}\left[\max _{0 \leq y \leq 1} z_{2 n-1}(x, y)+\min _{0 \leq y \leq 1} z_{2 n-1}(x, y)\right], \\
h_{n}(y) & =\frac{1}{2}\left[\max _{0 \leq x \leq 1} z_{2 n}(x, y)+\min _{0 \leq x \leq 1} z_{2 n}(x, y)\right] .
\end{aligned}
$$

It is obvious that $z_{n} \sim z(n=1,2, \cdots)$. The passage from $z_{2 n-1}$ to $z_{2 n}$ reduces $\left\|z_{2 n-1}\right\|$ by the maximal amount by which it can be reduced through the subtraction of a function of $x$, while the passage from $z_{2 n}$ to $z_{2 n+1}$ reduces $\left\|z_{2 n}\right\|$ by the maximal amount by which it can be reduced through the subtraction of a function of $y$. Thus, if we let

$$
M_{n}[z]=\left\|z_{n}\right\|
$$


then the $M_{n}$ form a nonincreasing sequence of nonnegative numbers, so that $\lim _{n \rightarrow \infty} M_{n}[z]=M[z]$ exists. We have the following obvious result.

LEMMA 3. The functional $\mu[z]$ satisfies $\mu[z] \leq M[z]$.

Our solution of problem $B$ will be a consequence of the following theorem.

THE OREM 2. The functional $\mu[z]$ satisfies $\mu[z]=M[z]$.

This, incidentally, will establish the fact that the functional $M[z]$ is invariant under equivalence. The direct proof of this fact might prove somewhat cumbersome.

Keeping in mind the results of Lemmas 2 and 3 we see that both Theorems 1 and 2 are consequences of the following result.

THE OREM 3. The functional $\pi[z]$ satisfies $\pi[z]=M[z]$.

Proof. We shall call a function $z$ horizontally level if

$$
\max _{0 \leq x \leq 1} z(x, y)=-\min _{0 \leq x \leq 1} z(x, y)
$$

for $0 \leq y \leq 1$, and we shall call it vertically level if

$$
\max _{0 \leq y \leq 1} z(x, y)=-\min _{0 \leq y \leq 1} z(x, y)
$$

for $0 \leq x \leq 1$. For the sake of brevity we shall use the symbol $M$ instead of $M[z]$.

There exists a number $N$ such that $M_{2} N<M+\epsilon$, where $\epsilon$ is a small positive number which is to be further determined later. We now perform the next $2 n$ steps of the leveling process on the function $z_{2} N$.

There exists a point $\left(x_{1}, y_{1}\right)$ such that

$$
z_{2 N+2 n}\left(x_{1}, y_{1}\right)=M_{2 N+2 n}=M+\delta, \quad \delta \geq 0 ;
$$

and since $z_{2 N+2 n}$ is vertically level there exists a point $\left(x_{2}, y_{2}\right)$ with $x_{2}=x_{1}$ such that

$$
z_{2 N+2 n}\left(x_{2}, y_{2}\right)=-M-\delta
$$

Hence we have

$$
\begin{aligned}
& z_{2 N+2 n-1}\left(x_{1}, y_{1}\right)-g_{N+n}\left(x_{1}\right)=M+\delta, \\
& z_{2 N+2 n-1}\left(x_{2}, y_{2}\right)-g_{N+n}\left(x_{2}\right)=-M-\delta ;
\end{aligned}
$$


and since $M_{2 N+2 n-1}<M+\epsilon$ and $x_{1}=x_{2}$ this implies

$$
\begin{array}{rll}
M+\epsilon-g_{N+n}\left(x_{1}\right)>M+\delta & \text { or } & g_{N+n}\left(x_{1}\right)<\epsilon-\delta, \\
-M-\epsilon-g_{N+n}\left(x_{1}\right)<-M-\delta & \text { or } & g_{N+n}\left(x_{1}\right)>\delta-\epsilon .
\end{array}
$$

We therefore have certainly

$$
-\epsilon<g_{N+n}\left(x_{1}\right)<\epsilon
$$

Thus

$$
\begin{aligned}
& z_{2 N+2 n-1}\left(x_{1}, y_{1}\right)=M+\delta+g_{N+n}\left(x_{1}\right)>M+\delta-\epsilon, \\
& z_{2 N+2 n-1}\left(x_{2}, y_{2}\right)=-M-\delta+g_{N+n}\left(x_{1}\right)<-M-\delta+\epsilon ;
\end{aligned}
$$

and since $z_{2 N+2 n-1}$ is horizontally level there exists a point $\left(x_{3}, y_{3}\right)$ with $y_{3}=y_{2}$ such that

$$
z_{2 N+2 n-1}\left(x_{3}, y_{3}\right)>M+\delta-\epsilon \text {. }
$$

By the same process as we applied to $g_{N+n}\left(x_{1}\right)$ we can now show that

$$
\begin{aligned}
& -2 \epsilon<h_{N+n-1}\left(y_{1}\right)<2 \epsilon, \\
& -2 \epsilon<h_{N+n-1}\left(y_{2}\right)<2 \epsilon ;
\end{aligned}
$$

hence

$$
\begin{aligned}
& z_{2 N+2 n-2}\left(x_{1}, y_{1}\right)>M+\delta-3 \epsilon, \\
& z_{2 N+2 n-2}\left(x_{2}, y_{2}\right)<-M-\delta+3 \epsilon, \\
& z_{2 N+2 n-2}\left(x_{3}, y_{3}\right)>M+\delta-3 \epsilon ;
\end{aligned}
$$

further, because $z_{2 N+2 n-2}$ is vertically level, there exists a point $\left(x_{4}, y_{4}\right)$ with $x_{4}=x_{3}$ such that

$$
z_{2 N+2 n-3}\left(x_{4}, x_{4}\right)<-M-\delta+3 \epsilon
$$

Repeating this process $2 n$ times we finally obtain a sequence of points $\left(x_{1}, y_{1}\right)$, $\cdots,\left(x_{2 n+1}, y_{2 n+1}\right)$, such that $x_{2 k}=x_{2 k-1}, y_{2 k+1}=y_{2 k}(k=1, \cdots, n)$ and 


$$
\begin{array}{lr}
z_{2 N}\left(x_{2 k-1}, y_{2 k-1}\right)>M+\delta-\left(2^{2 n-1}-1\right) \epsilon & (k=1, \cdots, n+1), \\
z_{2 N}\left(x_{2 k}, y_{2 k}\right)<-M-\delta+\left(2^{2 n-1}-1\right) \epsilon & (k=1, \cdots, n) .
\end{array}
$$

We complete the above sequence of points to form a permissible line by adding the point $\left(x_{2 n+2}, y_{2 n+2}\right)$ with $x_{2 n+2}=x_{2 n+1}, y_{2 n+2}=y_{1}$. If we construct the functional $\pi_{l}$ associated with this permissible line then we obtain

$$
\begin{aligned}
\left|\pi_{l}[z]\right| & =\left|\pi_{l}\left[z_{2 N}\right]\right|=\frac{1}{2 n+2}\left|\sum_{j=1}^{2 n+2}(-1)^{j} z_{2 N}\left(x_{j}, y_{j}\right)\right| \\
& >\frac{2 n+1}{2 n+2}\left[M+\delta-\left(2^{2 n-1}-1\right) \epsilon\right]-\frac{1}{2 n+2}\left|z_{2 N}\left(x_{2 n+2}, y_{2 n+2}\right)\right| \\
& >M+\delta-\left(2^{2 n-1}-1\right) \epsilon-\frac{2}{2 n+2}(M+\epsilon) .
\end{aligned}
$$

Since the choice of $\epsilon$ was independent of $n$, we can choose $\epsilon$ so that $\left(2^{2 n-1}\right) \epsilon$ $=\epsilon_{1} / 2$ where $\epsilon_{1}$ is an arbitrary small positive number. At the same time we can choose $n$ so large that

$$
\frac{M+\epsilon}{n+1}<\frac{\epsilon_{1}}{2} \text {. }
$$

Thus we have: For every $\epsilon_{1}>0$ there exists a permissible line such that

$$
\left|\pi_{l}[z]\right|>M[z]-\epsilon_{1},
$$

or, in other words,

$$
\pi[z] \geq M[z]
$$

In conjunction with Lemmas 2 and 3 , this proves Theorem 3.

4. The discontinuous case. Examining our method of proof we can make the following observations :

(1) No essential use was made of the continuity of any of the functions $z(x, y), g(x), h(y)$ involved in the definition of $\mu[z]$. Specifically we may define

$$
\mu^{*}[z]=\inf _{g, h} \sup _{0 \leq x \leq 1,0 \leq y \leq 1}|z(x, y)-g(x)-h(y)|,
$$


where $z$ is an arbitrary (bounded) function defined for $0 \leq x \leq 1,0 \leq y \leq 1$ and $g(x), h(y)$ are arbitrary functions defined over $0 \leq x \leq 1$ and $0 \leq y \leq 1$, respectively. The definition of $\pi[z]$ remains valid for discontinuous $z$, while $M[z]$ can be extended to a functional $M *[z]$ which is defined for discontinuous (bounded) $z$, simply by replacing all the max and min symbols in the leveling process by sup and inf symbols respectively. With very minor modifications of the proof of Theorem 3 we then obtain the following result.

THEOREM $3 *$. The functional $\pi[z]$ satisfies $\pi[z]=\mu^{*}[z]=M^{*}[z]$, where (unless we wish to allow infinite values for these functionals) $z$ is an arbitrary bounded function.

Theorems 3 and $3^{*}$ yield the following corollary.

COROLLARY. If $z$ is continuous, then

$$
\mu[z]=\mu^{*}[z] .
$$

In other words, the approximation of a continuous $z(x, y)$ cannot be improved by permitting discontinuous $g(x)+h(y)$.

(2) The functions $\pi_{l}[z]$ are continuous functionals in our metric; more specifically, we have the following result.

Lемма 4. If $\|z-\bar{z}\|<\epsilon$, then $\left|\pi_{l}[z]-\pi_{l}[\bar{z}]\right|<\epsilon$ for any permissible line.

Proof. We have

$$
\begin{aligned}
& \pi_{l}[z]=\frac{1}{2 n} \sum_{j=1}^{2 n}(-1)^{j} z\left(x_{j}, y_{j}\right), \\
& \pi_{l}[\bar{z}]=\frac{1}{2 n} \sum_{j=1}^{2 n}(-1)^{j} \bar{z}\left(x_{j}, y_{j}\right) .
\end{aligned}
$$

Hence

$$
\begin{aligned}
\left|\pi_{l}[z]-\pi_{l}[\bar{z}]\right|=\frac{1}{2 n} \mid \sum_{j=1}^{2 n} & (-1)^{j}\left[z\left(x_{j}, y_{j}\right)-z\left(x_{j}, y_{j}\right)\right] \mid \\
& \leq \frac{1}{2 n} \sum_{j=1}^{2 n}\|z-\bar{z}\|<\frac{1}{2 n} \cdot 2 n \epsilon=\epsilon .
\end{aligned}
$$


As a consequence, $\pi[z]$ is itself a continuous functional, as expressed in the following corollary.

COROLLARY. If $\|z-\bar{z}\|<\epsilon$, where $z$ and $\bar{z}$ are arbitrary bounded functions, then

$$
|\mu *[z]-\mu *[\bar{z}]|<\epsilon
$$

It is also easy to show that the functionals $M_{n}^{*}[z]$ and $M_{n}^{*}[z]$ which arise in the leveling process are continuous in a similar sense.

5. The n-dimensional case. There was nothing in our treatment which demanded that $z$ be a function of two variables only, or even that the variables be numbers. Most generally we can say:

Let $S_{1}, S_{2}, \cdots, S_{k}$ be arbitrary point sets, and let $z(s)$ be a bounded function defined over the Cartesian product $S=S_{1} \times S_{2} \times \cdots \times S_{k}$. Let $T_{1}, T_{2}, \cdots$, $T_{l}$ be direct subproducts of the $S_{i}$ such that $T_{i} \nsubseteq T_{j}$ unless $i=j$. Let $E_{1}, E_{2}$, $\cdots, E_{l}$ be the projections of $S$ on $T_{1}, T_{2}, \cdots, T_{l}$ respectively. We now define

$$
\mu[z]=\inf _{f_{1}, \cdots, f_{l}} \sup _{s \in S}\left|z(s)-f_{1}\left(E_{1} s\right)-\cdots-f_{l}\left(E_{l} s\right)\right|,
$$

where $f_{i}$ ranges over all functions defined on $T_{i}$.

Our permissible lines are now replaced by a rather complicated permissible array of points $t_{i_{1}, i_{2}, \cdots, i_{m}}$ defined as follows :

(a) $t_{1}$ is an arbitrary point of $S$;

(b) to every point $t_{i_{1}}, \cdots, i_{m}$ there exist $l$ points $t_{i_{1}}, \cdots, i_{m} 1 ; t_{i_{1}}, \cdots, i_{m} 2 ; \cdots$; $t_{i_{1}, \cdots, i_{m}, l}$ such that $E_{j} t_{i_{1}, \cdots, i_{m}, j}=E_{j} t_{i_{1}, \cdots, i_{m}}$

(c)

$$
t_{i_{1}, \cdots, i_{m}, j, j}=t_{i_{1}, \cdots, i_{m}} ; t_{i_{1}, \cdots, j, i_{m}, j}=t_{i_{1}, \cdots, j} ;
$$

(d) the number of points in the set is finite;

(e) if $t_{i_{1}, \cdots, i_{m}}=t_{j_{1}}, \cdots, j_{n}$ then $m \equiv n(\bmod 2)$.

(This last condition is not really necessary but it serves to avoid confusion.)

In order to visualize these sets it might be well to consider the case where $S$ is three-dimensional Euclidean space; that is, $S_{1}$ is the $x$-axis, $S_{2}$ the $y$-axis, and $S_{3}$ the $z$-axis. If we take $T_{1}, T_{2}, T_{3}$ as the three coordinate planes then the permissible point sets consist of the vertices of closed polyhedral surfaces whose edges are parallel to the coordinate axes. If we take $T_{1}, T_{2}, T_{3}$ as the three coordinate axes then the permissible point sets consist of the vertices of closed polyhedral surfaces whose edges are parallel to the coordinate planes. 
To each permissible point set $p$ we now associate the functional

$$
\pi_{p}[z]=\frac{1}{N} \sum(-1)^{m} z\left(t_{i_{1}}, \cdots, i_{m}\right),
$$

where the summation is extended over all the $N$ different points of the permissible set. If we generalize the concept of equivalence so that $z \sim \bar{z}$ whenever

$$
z(s)-\bar{z}(s)=f_{1}\left(E_{1} s\right)+\cdots+f_{l}\left(E_{l} s\right),
$$

then $\pi_{l}[z]$ is seen to be invariant under equivalence.

The leveling process consists in the construction of the sequences

$$
z_{n}(s), f_{1}^{(n)}\left(E_{1} s\right), \cdots, f_{l}^{(n)}\left(E_{l} s\right)
$$

according to the following rules :

$$
\begin{aligned}
z_{1}(s)= & z(s), \quad z_{m l+j}(s)=z_{m l+j-1}(s)-f_{j}^{(m)}\left(E_{j} s\right), \\
f_{j}^{(m)}\left(E_{j} s\right)= & \frac{1}{2}\left[\sup _{E_{j} t=E_{j} s} z_{m l+j-1}(t)+\inf _{E_{j} t=E_{j} s} z_{m l+j-1}(t)\right] \\
& (j=1, \cdots, l ; n=0,1, \cdots) .
\end{aligned}
$$

We can again define the nonincreasing sequence of nonnegative functionals

$$
M_{n}[z]=\sup _{s \in S}\left|z_{n}(s)\right|
$$

and

$$
M[z]=\lim _{n \rightarrow \infty} M_{n}[z] .
$$

All the above lemmas and theorems remain valid under these new definitions; and the proofs, while more difficult to state, contain essentially no new ideas. Probably the greatest deviation from the above proofs takes place in the construction of the permissible set through the leveling process in the proof of Theorem 3. We shall therefore describe that process in greater detail.

Choose $N$ so large that $M_{N l+1}<M+\epsilon$, and let $M_{N l+n l+1}=M+\delta$; then there exist two points $t_{1}$ and $t_{4}$ in $S$ such that $E_{1} t_{1}=E_{1} t_{1,1}$, and

$$
\begin{gathered}
z_{N l+n l+1}\left(t_{1}\right)>M-\epsilon, \\
z_{N l+n l+1}\left(t_{1,1}\right)<-M+\epsilon .
\end{gathered}
$$


and that there exist points $t_{1,2}$ and $t_{1,1,2}$ in $S$ such that $E_{2} t_{1}=E_{2} t_{1,2} ; E_{2} t_{1,1}$ $=E_{2} t_{1,1,2}$, and

$$
\begin{aligned}
& z_{N l+n l}\left(t_{1,2}\right)<-M+3 \epsilon, \\
& z_{N l+n l}\left(t_{1,1,2}\right)>M-3 \epsilon .
\end{aligned}
$$

The next step in the leveling process adds the points $t_{1,3}, t_{1,1,3}, t_{1,2,3}$,

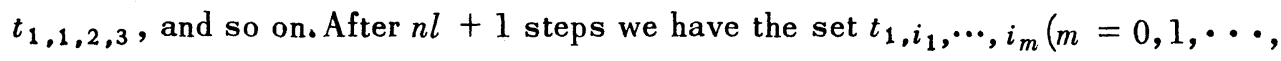
$\left.n l ; i_{j} \in\{1,2, \cdots, l\}\right)$, where

$$
\begin{gathered}
z_{N l}\left(t_{1, i_{1}, \cdots, i_{2 m}}\right)>M-\left(2^{n l+2}-1\right) \epsilon, \\
z_{N l}\left(t_{1, i_{1}, \cdots, i_{2 m+1}}\right)<-M+\left(2^{n l+2}-1\right) \epsilon .
\end{gathered}
$$

In order to form a permissible point set we have to adjoin additional points so that condition (b) will be satisfied. Condition (b) is already satisfied for all points $t_{1, i_{1}}, \cdots, i_{m}$ with $m \leq(n-1) l$. The number of points with $(n-1) l<m \leq n l$ is $l !$, and is therefore independent of $n$. It is easy to see that by adding a fixed finite number of points (this number $A$ depends on $k$ and $l$ but not on $n$ ) we can obtain a point set which satisfies condition (b). Thus the augmented point set satis-

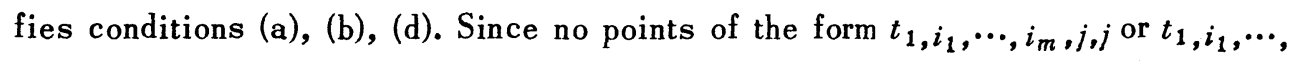
$j, i_{m}, j$ were constructed in the leveling process we can satisfy condition (c) by definition. For the nonaugmented point set, condition (c) is an immediate consequence of (1) if $\epsilon$ is sufficiently small. The augmented part can be constructed so that (c) is satisfied. We denote the nonaugmented set by $p^{\prime}$, the augmenting set by $p^{\prime \prime}$.

Thus we have constructed a permissible point set; if we form the corresponding functional $\pi_{p}[z]$, then we have

$$
\begin{aligned}
\left|\pi_{p}[z]\right| & =\frac{1}{B}\left|\sum_{p}(-1)^{m} z_{N l}\left(t_{1, i_{1}, \cdots, i_{m}}\right)\right| \\
& \geq \frac{1}{B}\left|\sum_{p^{\prime}}(-1)^{m} z_{N l}\left(t_{1, i_{1}, \cdots, i_{m}}\right)\right|-\frac{1}{B}\left|\sum_{p^{\prime \prime}} z_{N l}\left(t_{\left.1, i_{1}, \cdots, i_{m}\right)}\right)\right| \\
& \geq \frac{1}{B}(B-A)\left[M-\left(2^{n l+2}-1\right) \epsilon\right]-\frac{1}{B} A(M+\epsilon)
\end{aligned}
$$




$$
=M-\frac{2 A}{B} M+\frac{B-A}{B}\left[\left(2^{n l+2}-1\right)-\frac{A}{B}\right] \epsilon,
$$

where $B=B(n)$ is the number of points in the permissible set, so that $B(n) \rightarrow \infty$ with $n$. For a suitable choice of $\epsilon$ and $n$ we have

$$
\left|\pi_{p}[z]\right| \geq M-\epsilon .
$$

Hence Theorem 3 is true in this generalized case.

6. Further discussion of the leveling process. While the leveling process gave rise to a sequence of functions $z_{n} \sim z$ with $\lim _{n \rightarrow \infty}\left\|z_{n}\right\|=\mu[z]$, we were unable to show the convergence of the functions $z_{n}$. In fact, we have not yet proved the existence of a function $\bar{z} \in C_{R}$ with $\bar{z} \sim z$ and $\|\bar{z}\|=\mu[z]$, nor did we investigate the rate of convergence of $\left\|z_{n}\right\|$. It is the purpose of this section to treat the last two questions.

In order to prove the existence of $\bar{z}$ we prove the equicontinuity of the sequence $\left\{z_{n}\right\}$ and thus insure the existence of a convergent subsequence with a continuous limit $\bar{z}$. To this end we first prove:

Lemma 5. If $f_{1}(x), f_{2}(x) \in C_{x}$ and $\left\|f_{1}-f_{2}\right\|<\epsilon$, then

$$
\left|\max _{0 \leq x \leq 1} f_{1}(x)-\max _{0 \leq x \leq 1} f_{2}(x)\right|<\epsilon \text { and }\left|\min _{0 \leq x \leq 1} f_{1}(x)-\min _{0 \leq x \leq 1} f_{2}(x)\right|<\epsilon .
$$

Proof. Since

$$
\min _{0 \leq x \leq 1} f(x)=-\max _{0 \leq x \leq 1}[-f(x)],
$$

it suffices to prove the first statement. Let

$$
f_{1}\left(x_{1}\right)=\max _{0 \leq x \leq 1} f_{1}(x)
$$

then

and hence

$$
f_{2}\left(x_{1}\right)>f_{1}\left(x_{1}\right)-\epsilon=\max _{0 \leq x \leq 1} f_{1}(x)-\epsilon,
$$

$$
\max _{0 \leq x \leq 1} f_{2}(x)>\max _{0 \leq x \leq 1} f_{1}(x)-\epsilon,
$$


or

$$
\max _{0 \leq x \leq 1} f_{1}(x)-\max _{0 \leq x \leq 1} f_{2}(x)<\epsilon
$$

Similarly,

$$
\max _{0 \leq x \leq 1} f_{2}(x)-\max _{0 \leq x \leq 1} f_{1}(x)<\epsilon
$$

We define

$$
\begin{aligned}
& \bar{g}_{n}(x)=\sum_{k=1}^{n} g_{k}(x), \\
& \bar{h}_{n}(y)=\sum_{k=1}^{n} h_{k}(y),
\end{aligned}
$$

so that

$$
z_{2 n+1}=z-\bar{g}_{n}-\bar{h}_{n}
$$

The equicontinuity of $\left\{z_{n}\right\}$ will be the direct consequence of the following result.

THEOREM 4. If for fixed $y$ and $\Delta y$ we have

$$
|z(x, y)-z(x, y+\Delta y)|<\epsilon \text { for } 0 \leq x \leq 1 \text {, }
$$

then we have

$$
\left|\bar{h}_{n}(y)-\bar{h}_{n}(y+\Delta y)\right|<\epsilon \quad(n=1,2, \cdots) .
$$

Similarly, if for fixed $x$ and $\Delta x$ we have

$$
|z(x, y)-z(x+\Delta x, y)|<\epsilon \quad \text { for } \quad 0 \leq y \leq 1,
$$

then we have

$$
\left|\bar{g}_{n}(x)-\bar{g}_{n}(x+\Delta x)\right|<\epsilon \quad(n=1,2, \cdots) .
$$

Proof. It obviously suffices to prove the first part of the theorem.

Let $z^{*}=z(x, y)-\bar{g}_{n}(x)$; then

$\left|z^{*}(x, y)-z^{*}(x, y+\Delta y)\right|=|z(x, y)-z(x, y+\Delta y)|<\epsilon \quad$ for $\quad 0 \leq x \leq 1$. 
Hence, if in Lemma 5 we let $f_{1}(x)=z^{*}(x, y)$ and $f_{2}(x)=z^{*}(x, y+\Delta y)$, we obtain

$$
\begin{aligned}
& \left|\max _{0 \leq x \leq 1}\left[z(x, y)-\bar{g}_{n}(x)\right]-\max _{0 \leq x \leq 1}\left[z(x, y+\Delta y)-\bar{g}_{n}(x)\right]\right|<\epsilon, \\
& \left|\min _{0 \leq x \leq 1}\left[z(x, y)-\bar{g}_{n}(x)\right]-\min _{0 \leq x \leq 1}\left[z(x, y+\Delta y)-\bar{g}_{n}(x)\right]\right|<\epsilon .
\end{aligned}
$$

Now $z_{2 n+1}=z-\bar{g}_{n}-\bar{h}_{n}$ is vertically level. Hence

$$
\begin{aligned}
\bar{h}_{n}(y) & =\frac{1}{2}\left\{\max _{0 \leq x \leq 1}\left[z(x, y)-\bar{g}_{n}(x)\right]+\min _{0 \leq x \leq 1}\left[z(x, y)-\bar{g}_{n}(x)\right]\right\}, \\
\bar{h}_{n}(y+\Delta y) & =\frac{1}{2}\left\{\max _{0 \leq x \leq 1}\left[z(x, y+\Delta y]-\bar{g}_{n}(x)\right]+\min _{0 \leq x \leq 1}\left[z(x, y+\Delta y)-\bar{g}_{n}(x)\right]\right\},
\end{aligned}
$$

or

$$
\begin{aligned}
\mid \bar{h}_{n}(y) & -\bar{h}_{n}(y+\Delta y) \mid \\
& \leq \frac{1}{2}\left|\max _{0 \leq x \leq 1}\left[z(x, y)-\bar{g}_{n}(x)\right]-\max _{0 \leq x \leq 1}\left[z(x, y+\Delta y)-\bar{g}_{n}(x)\right]\right| \\
& +\frac{1}{2}\left|\min _{0 \leq x \leq 1}\left[z(x, y)-\bar{g}_{n}(x)\right]-\min _{0 \leq x \leq 1}\left[z(x, y+\Delta y)-\bar{g}_{n}(x)\right]\right|<\epsilon .
\end{aligned}
$$

The discussion so far has failed to settle the questions of the rate of convergence of $\left\|z_{n}\right\|$ and of the convergence of $z_{n}$. We were able to obtain only partial answers. At the suggestion of the referee we omit the proofs of most of the following statements; their sequence will have to indicate our derivation.

Lemma 6. For $n \geq 2$ we have

$$
\left\|g_{n}\right\| \geq\left\|h_{n}\right\| \geq\left\|g_{n+1}\right\| \geq\left\|h_{n+1}\right\|>\cdots \text {. }
$$

Lemma 7. We have also 


$$
\begin{aligned}
\left\|z_{2 n-1}\right\| & \geq-\left\|z_{2 n+2 N-1}\right\|+\left(2^{2 N+1}-2\right)\left\|g_{N+n}\right\| \\
& -\sum_{k=1}^{N}\left(2^{2 N-2 k+1}-1\right)\left\|g_{N+n-k}\right\|-\sum_{k=1}^{N}\left(2^{2 N-2 k+2}-1\right)\left\|h_{N+n-k}\right\| .
\end{aligned}
$$

THЕОВем 5. The norm $\|z\|$ satisfies

$$
\|z\| \geq N\left\|g_{n}\right\|-\left(2^{2 n}-1\right)\left(\left\|g_{n}\right\|-\left\|g_{N+n}\right\|\right) \text {. }
$$

THЕОRЕм 6. For every $\epsilon>0$ there is an $n_{0}$ such that for all $n>n_{0}$ we have

$$
\left\|g_{n}\right\|<(2+\epsilon)\|z\| / \log _{2} n \text {. }
$$

COROLLARY. The following relations hold:

$$
\lim _{n \rightarrow \infty}\left\|g_{n}\right\|=\lim _{n \rightarrow \infty}\left\|h_{n}\right\|=0 \text {. }
$$

Definition. A function $z(x, y)$ is level if it is both horizontally and vertically level.

THEовем 7. For every $z \in C_{R}$ there is a $z \in C_{R}$ such that $z$ is level, $\bar{z} \sim z$, and $\mu[z]=\|\bar{z}\|$.

Proof. According to Theorem 4, the sequence $\left\{z_{n}\right\}$ has a uniformly convergent subsequence $\left\{z_{n_{i}}\right\}$. Let

$$
\bar{z}=\lim _{i \rightarrow \infty} z_{n_{i}} .
$$

According to Theorem 6 we have

$$
\lim _{i \rightarrow \infty}\left\|z_{n_{i}+1}-z_{n_{i}}\right\|=0
$$

hence

$$
\bar{z}=\lim _{i \rightarrow \infty} z_{n_{i}+1} \text {. }
$$

Of the two functions $z_{n_{i}}, z_{n_{i+1}}$ one is horizontally level, while the other is vertically level; hence the common uniform limit is level.

Since $z_{n_{i}} \sim z$, we have $\bar{z} \sim z$ and

$$
\|\bar{z}\|=\lim _{i \rightarrow \infty}\left\|z_{n_{i}}\right\|=\lim _{n \rightarrow \infty}\left\|z_{n}\right\|=\mu[z] .
$$


We remark that the bound obtained for $\left\|g_{n}\right\|$ in Theorem 6 .does not seem to be the best possible. In fact in all the cases we have investigated we obtained $\left\|g_{n}\right\|<c 2^{-n}$. Such an estimate would of course settle the unsolved question of the convergence of the sequence $\left\{z_{n}\right\}$.

Another unsettled question is that of the existence of a minimizing function $\bar{z}$ equivalent to a discontinuous function $z$. While Theorems $4-7$ remain valid with minor modifications for discontinuous $z$, Theorem 4 no longer implies the existence of a convergent subsequence of $\left\{z_{n}\right\}$.

UNIVERSITY OF CALIF ORNIA, BERKELEY

University of California, Los Angeles 


\title{
PACIFIC JOURNAL OF MATHEMATICS
}

\section{EDITORS}

\author{
Herbert BuSEMANN \\ R. M. RoBINSON \\ University of Southern California \\ University of California \\ Los Angeles 7, California \\ Berkeley 4, California \\ E. F. BEC KENBACH, Managing Editor \\ University of California \\ Los Angeles 24, California
}

\section{ASSOCIATE EDITORS}
R. P. DILWORTH
P. R. HALMOS
BØRGE JESSEN
J. J. STOKER
HERBERT FEDERER
HEINZ HOPF
PAUL LÉVY
MARSHALL HALL
R. D. JAMES
GEORGE PÓLYA
E. G. STRAUS
KÖSAKU YOSIDA

\section{SPONSORS}

UNIVERSITY OF BRITISH COLUMBIA

CALIFORNIA INSTITUTE OF TECHNOLOGY

UNIVERSITY OF CALIFORNIA, BERKELEY

UNIVERSITY OF CALIFORNIA, DAVIS

UNIVERSITY OF CALIFORNIA, LOS ANGELES

UNIVERSITY OF CALIFORNIA, SANTA BARBARA

OREGON STATE COLLEGE

UNIVERSITY OF OREGON
UNIVERSITY OF SOUTHERN CALIFORNIA

STANFORD UNIVERSITY

WASHINGTON STATE COLLEGE

UNIVERSITY OF WASHINGTON

AMERICAN MATHEMATICAL SOCIETY

NATIONAL BUREAU OF STANDARDS, INSTITUTE FOR NUMERIGAL ANALYSIS

Mathematical papers intended for publication in the Pacific Journal of Mathematics should be typewritten (double spaced), and the author should keep a complete copy. Manuscripts may be sent to any of the editors. All other communications to the editors should be addressed to the managing editor, E. F. Beckenbach, at the address given above.

Authors are entitled to receive 100 free reprints of their published papers and may obtain additional copies at cost.

The Pacific Journal of Mathematics is published quarterly, in March, June, September, and December. The price per volume (4 numbers) is $\$ 8.00$; single issues, $\$ 2.50$. Spécial price to individual faculty members of supporting institutions and to members of the American Mathematical Society: $\$ 4.00$ per volume; single issues, $\$ 1.25$.

Subscriptions, orders for back numbers, and changes of address should be sent to the publishers, University of California Press, Berkeley 4, California.

UNIVERSITY OF CALIFORNIA PRESS - BERKELEY AND LOS ANGELES 


\section{Pacific Journal of Mathematics}

\section{Vol. 1, No. $2 \quad$ December, 1951}

Tom M. (Mike) Apostol, On the Lerch zeta function ................. 161

Ross A. Beaumont and Herbert S. Zuckerman, A characterization of the subgroups of the additive rationals ....................... 169

Richard Bellman and Theodore Edward Harris, Recurrence times for the Ehrenfest model................................... 179

Stephen P.L. Diliberto and Ernst Gabor Straus, On the approximation of a function of several variables by the sum of functions of fewer

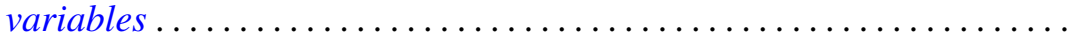

Isidore Isaac Hirschman, Jr. and D. V. Widder, Convolution transforms with complex kernels ................................ 211

Irving Kaplansky, A theorem on rings of operators .............. 227

W. Karush, An iterative method for finding characteristic vectors of a symmetric matrix............................... 233

Henry B. Mann, On the number of integers in the sum of two sets of positive integers ......................................... 249

William H. Mills, A theorem on the representation theory of Jordan

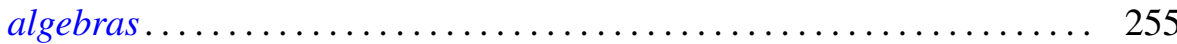

Tibor Radó, An approach to singular homology theory.............. 265

Otto Szász, On some trigonometric transforms ................... 291

James G. Wendel, On isometric isomorphism of group algebras ......... 305

George Milton Wing, On the $L^{p}$ theory of Hankel transforms ... 